\title{
Theranosties
}

Editorial

2011; 1:1-2

\section{Introducing Theranostics Journal - From the Editor-in-Chief}

\author{
Xiaoyuan (Shawn) Chen ${ }^{凶}$ \\ Laboratory of Molecular Imaging and Nanomedicine, National Institute of Biomedical Imaging and Bioengineering, Na- \\ tional Institutes of Health, Maryland 20892, USA \\ $\bowtie$ Corresponding author: Shawn.Chen@nih.gov \\ (C) Ivyspring International Publisher. This is an open-access article distributed under the terms of the Creative Commons License (http://creativecommons.org/ \\ licenses/by-nc-nd/3.0/). Reproduction is permitted for personal, noncommercial use, provided that the article is in whole, unmodified, and properly cited.
}

Published: 2011.01 .08

\begin{abstract}
Theranostics is a multidisciplinary journal that publishes innovative and original research papers reflecting the field of molecular imaging, molecular therapeutics, multifunctional nanoparticle platforms, image-guided therapy, and translational nanomedicine. A broad spectrum of biomedical research that can be applied to future theranostic applications is encouraged.
\end{abstract}

Key words: molecular imaging, molecular therapy, theranostics, nanomedicine

It is my great pleasure to introduce the new journal Theranostics. Theranostics is a concept that was originally raised to refer to the efforts of integrating imaging and therapy. As an emerging interdiscipline, it is related to but different from traditional imaging and therapeutics. It embraces the efforts of utilizing multiple techniques to arrive at a comprehensive imaging/therapy regimen. More recently, there is a trend of tangling those efforts with emerging materials science and nanotechnologies, in an attempt to develop novel platforms and methodologies to tackle practical issues in clinics. Work in this area can be significant for being cutting-edge and potentially translatable, and the community has grown up rapidly.

Unfortunately, most existing journals in the fields of imaging or therapy, such as Journal of Nuclear Medicine, Magnetic Resonance Imaging and Radiology, etc., focus more on single modality imaging and do not necessarily encourage comprehensive theranostic studies. On the other hand, materials based journals, such as Nano Letters, SMALL and ACS Nano, have diversified interests in nanotechnologies, and are not particularly addressing the clinical relevance of the research work. Launching a journal on this topic is therefore significant for filling the gap and promoting the concept of theranostics.
The aim of Theranostics is to provide a forum for the exchange of clinical and scientific information for the diagnostic and therapeutic molecular and nano medicine community and allied professions involved in the efforts of integrating molecular imaging and molecular therapy. Theranostics is a multidisciplinary journal that publishes innovative and original research papers reflecting the field of molecular imaging, molecular therapeutics, multifunctional nanoparticle platforms, image-guided therapy, and translational nanomedicine. A broad spectrum of biomedical research that can be applied to future theranostic applications is encouraged. In addition to primary research, Theranostics also publishes reviews, news, and commentary to understand and bridge the gap among the laboratory, clinic, and biotechnology industries.

Note that in some cases theranostics is also spelled as theragnostics. Keyword search of PubMed as of January 7, 2010 found 95 items for "theranostic", 63 items for "theranostics", 29 items for "theragnostic" and 21 items for "theragnostics".

This journal will be published using an Open Access publishing model, which means that accepted papers will be freely and immediately available on the journal's website without any access barriers. It is our belief that the Open Access model will become a major force in scientific publishing in the near future, and 
we hope that Theranostics will be a leading journal in this new movement.

In both the review process and the production process we will aim for the highest possible quality and speed. Papers in the journal will be rigorously peer-reviewed. We have already assembled a first-rate Editorial Board, and we plan to continuously strengthen it in terms of academic authority, specialty balance, geographic scope, junior involvement, and individual productivity. Each board member will make active contributions and be evaluated yearly with routine adjustment for maximum benefit of the journal. Special Issues will be organized to make well defined contributions to the literature in key areas of research.

In opening up this new journal, we are very pleased and thankful to receive the brilliant contribu-

\section{Author biography}

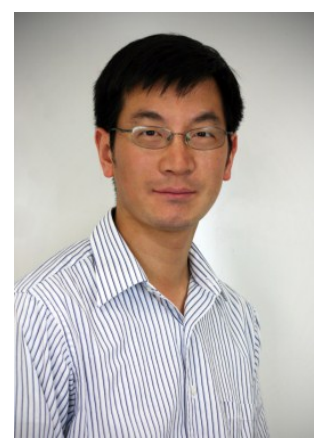

Xiaoyuan (Shawn) Chen received his B.S. in 1993 and M.S. in 1996 from Nanjing University, China. He then moved to the states and obtained his Ph.D. in chemistry from the University of Idaho in 1999. After two quick postdocs at Syracuse University and Washington University in St. Louis, he joined the University of Southern California as an Assistant Professor of Radiology. He then moved to Stanford University in 2004 to help build up the Molecular Imaging Program at Stanford (MIPS) under the directorship of Prof. Sanjiv Sam Gambhir. He was promoted to Associate Professor in 2008 and in the summer of 2009, he joined the intramural research program of the National Institute of Biomedical Imaging and Bioengineering (NIBIB) as a tenured senior investigator and lab chief. He took over the Positron Emission Tomography Radiochemistry Group and expanded it into the Laboratory of Molecular Imaging and Nanomedicine (LOMIN). He tions and supports from many distinguished authors and organizations all over the world. I thank the pioneer authors, all members of the founding Editorial Board, and members of publishing team in Ivyspring International Publisher for their efforts in providing significant supports and suggestions and organizing the Theranostics from ground zero.

We are confident that Theranostics will soon establish itself as a highly reputable journal, due to the fact that it is the first journal in this important and emerging field, the advantages of its Open Access publishing model, comprehensive coverage, novel features, and high academic standards. Please feel free to contact the Editor-in-Chief if you have any ideas or suggestions.

also has joint appointments with the NIH Clinical Center and National Institute of Standards and Technologies (NIST). Dr. Chen has published over 250 papers and numerous books and book chapters. $\mathrm{He}$ sits on the editorial board of over 10 peer-reviewed journals and is a regular reviewer for over 50 journals. The Laboratory of Molecular Imaging and Nanomedicine (LOMIN) specializes in synthesizing molecular imaging probes for positron emission tomography (PET), single-photon emission computed tomography (SPECT), magnetic resonance imaging (MRI), optical (bioluminescence, fluorescence and Raman), contrast enhanced ultrasound, photoacoustic imaging as well as multimodality imaging. LOMIN aims to develop molecular imaging toolbox for better understanding of the biology, early diagnosis of diseases, monitoring therapy response, and guiding drug discovery/development. There is a special emphasis of LOMIN on high sensitivity nanosensors for biomarker detection and theranostic nanomedicine for imaging, gene and drug delivery, and monitoring of treatment. 